\title{
Equilibrium temperature distribution and Hadley circulation in an axisymmetric model
}

\author{
N. Tartaglione \\ School of Science and Technology, University of Camerino, Camerino, Italy \\ Correspondence to: N. Tartaglione (nazario.tartaglione@unicam.it)
}

Received: 5 October 2014 - Published in Nonlin. Processes Geophys. Discuss.: 3 November 2014

Revised: 8 January 2015 - Accepted: 20 February 2015 - Published: 16 March 2015

\begin{abstract}
The impact of the equilibrium temperature distribution, $\theta_{\mathrm{E}}$, on the Hadley circulation simulated by an axisymmetric model is studied. The $\theta_{\mathrm{E}}$ distributions that drive the model are modulated here by two parameters, $n$ and $k$, the former controlling the horizontal broadness and the latter controlling the vertical stratification of $\theta_{\mathrm{E}}$. In the present study, variations in the $\theta_{\mathrm{E}}$ distribution mimic changes in the energy input of the atmospheric system, leaving as almost invariant the Equator-poles $\theta_{\mathrm{E}}$ difference. Both equinoctial and time-dependent Hadley circulations are simulated and the results compared. The results give evidence that concentrated $\theta_{\mathrm{E}}$ distributions enhance the meridional circulation and jet wind speed intensities, even with a lower energy input. The meridional circulation and the subtropical jet stream widths are controlled by the broadness of horizontal $\theta_{\mathrm{E}}$ rather than by the vertical stratification, which is important only when $\theta_{\mathrm{E}}$ distribution is concentrated at the Equator. The jet stream position does not show any dependence with $n$ and $k$, except when the $\theta_{\mathrm{E}}$ distribution is very wide $(n=3)$ and, in such a case, the jet is located at the mid-latitudes and the model temperature clamps to forcing $\theta_{\mathrm{E}}$. Using $n=2$ and $k=1$, we have the formulation of the potential temperature adopted in the classical literature. A comparison with other works is performed, and our results show that the model running in different configurations (equinoctial, solstitial and time dependent) yields results similar to one another.
\end{abstract}

\section{Introduction}

The Earth's atmosphere is driven by differential heating of the Earth's surface. At the Equator, where the heating is larger than that at other latitudes, air rises and diverges pole- ward in the upper troposphere, descending more or less at $30^{\circ}$ latitude. This meridional circulation is known as the Hadley cell. Two subtropical jets at the poleward edges of the Hadley cell form because of the Earth's rotation and the conservation of the angular momentum. A poleward shift (Fu and Lin, 2011) and an enhanced wind speed of these jets (Strong and Davis, 2007) are associated with possible Hadley cell widening and strengthening, which has been observed in the last decades (Fu et al., 2006; Hu and Fu, 2007; Seidel et al., 2008; Johanson and $\mathrm{Fu}, 2009$; Nguyen et al., 2013).

There are a few studies suggesting possible causes of these phenomena. One of the theories postulates global warming as a possible cause of Hadley cell widening (Lu et al., 2009). However, the atmosphere is a complex system containing many subsystems interacting with one another, and global warming might not be the only cause that is suggested to explain the widening. Ozone depletion (Lu et al., 2009; Polvani et al., 2011), SST warming (Chen et al., 2013; Staten et al., 2011) and aerosol (Allen et al., 2012) have also been invoked to explain the Hadley cell widening.

Climate models vary to some extent in their response and the relationship between global warming and the Hadley cell is not straightforward. For instance, Lu et al. (2007) found a smaller widening than the observed one. Gitelman et al. (1997) showed that the meridional temperature gradient decreases with increasing global mean temperature, and the same result can be found in recent modeling studies (Schaller et al., 2013).

Much of our understanding of the Hadley cell comes from theories using simple models (Schneider, 1977; Schneider and Lindzen, 1977; Held and Hou, 1980, hereafter HH80), and such a simple model will be adopted here in order to understand how temperature distributions can change the 
Hadley circulation. How much temperature change impacts the real Hadley circulation is not clear yet, perhaps because of discrepancies between observations, reanalysis (Waliser et al., 1999) and climate model outputs, although these differences are becoming less marked because of newer observational data sets or corrections of the older ones (Sherwood et al., 2008; Titchner et al., 2008; Santer et al., 2008). Hence, it is critical to understand the possible mechanisms behind the cell expansion starting from a simple model.

The objective of this study is to analyze the sensitivity of a model of symmetric circulation to the radiative-convective equilibrium temperature distribution. Our point of departure is the symmetric model used by Cessi (1998), which is a bidimensional model considering atmosphere as a thin spherical shell. This model will be briefly described in Sect. 2 . The model mainly describes a tropical atmosphere; hence, it does not allow for eddies. Although eddies may play a central role in controlling the strength and width of the Hadley cell (e.g., Kim and Lee, 2001; Walker and Schneider, 2006), a symmetric circulation, driven by latitudinal differential heating, can exist even without eddies, and it is a robust feature of the atmospheric system (Dima and Wallace, 2003). The temperature distributions used in this study represent some paradigms of tropical atmospheres. Among the possible causes that can change temperature distributions are El Niño, global warming and changes in solar activity. We will show, in Sect. 3, that the energy input is not as important as the forcing distribution. Our results are consistent with those obtained both by Hou and Lindzen (1992) (hereafter HL92) and recently by Tandon et al. (2013), who performed experiments similar to those described here. The conclusions will be drawn in Sect. 4.

\section{The model}

The model used in this study is a bi-dimensional model of the axisymmetric atmospheric circulation described in Cessi (1998). The horizontal coordinate is defined as $y=a \sin \phi$, from which we have

$c(y)=\cos \phi=\sqrt{\left(1-y^{2} / a^{2}\right)}$,

where $a$ is the radius of a planet having a rotation rate $\Omega$; the height of the atmosphere is prescribed to be $H$.

The model is similar to the Held and Hou model (HH80), but it prescribes a horizontal viscosity $v_{\mathrm{H}}$ other than the vertical viscosity $v_{\mathrm{v}}$. The prognostic variables are the angular momentum $M$, defined as $M=\Omega a c^{2}+u c$, where $u$ represents the zonal velocity; the zonal vorticity $\psi_{z z}$ with the meridional stream function $\psi$ is defined by

$\partial_{y} \psi \equiv w$,

$\partial_{z} \psi \equiv-c v$,

and the potential temperature $\theta$ that is forced towards a radiative-convective equilibrium temperature $\theta_{\mathrm{E}}$. Starting from the dimensional equations of the angular momentum, zonal vorticity and potential temperature, we will obtain a set of dimensionless equations. The new equations are nondimensionalized using a scaling that follows Schneider and Lindzen (1977), but the zonal velocity $u$ is scaled with $\Omega a$. A detailed description can be found in Cessi (1998).

The non-dimensional model equations are

$$
\begin{aligned}
M_{t}= & \frac{1}{R}\left\{M_{z z}+\mu\left[c^{4}\left(c^{-2} M\right)_{y}\right]_{y}\right\}-J(\psi, M), \\
\psi_{z z t}= & \frac{1}{\left(R^{2} E^{2}\right)} y c^{-2}\left(M^{2}\right)_{z}-\frac{1}{c^{-2}} J\left(\psi, c^{-2} \psi_{z z}\right) \\
& +\frac{1}{\left(R E^{2} c^{-2}\right)} \theta_{y}+\frac{1}{\left(R c^{-2}\right)}\left[c^{-2} \psi_{z z z z}+\mu \psi_{z z y y}\right], \\
\theta_{t}= & \frac{1}{R}\left\{\theta_{z z}+\mu\left[c^{2} \theta_{y}\right]_{y}+\alpha\left[\theta_{\mathrm{E}}(y, z)-\theta\right]\right\}-J(\psi, \theta) .
\end{aligned}
$$

The term $J(A, B)=A_{y} B_{z}-A_{z} B_{y}$ is the Jacobian.

The thermal Rossby number $R$, the Ekman number $E$, the ratio of the horizontal to the vertical viscosity $\mu$ and the parameter $\alpha$ are defined as

$$
\begin{aligned}
& R \equiv g H \Delta_{\mathrm{H}} /\left(\Omega^{2} a^{2}\right) ; E \equiv \nu_{\mathrm{V}} /\left(\Omega H^{2}\right) ; \\
& \mu \equiv\left(H^{2} / a^{2}\right) \nu_{\mathrm{H}} / \nu_{\mathrm{V}} ; \alpha \equiv H^{2} /\left(\tau \nu_{\mathrm{V}}\right) .
\end{aligned}
$$

The term $\alpha$ is the ratio of the viscous timescale across the depth of the model atmosphere to the relaxation time $\tau$ toward the radiative-convective equilibrium.

The boundary conditions for the set of Eq. (3) are

$$
\begin{aligned}
& M_{z}=\gamma\left(M-c^{2}\right), \psi_{z z}=\gamma \psi_{z}, \\
& \psi=\theta_{z}=0 \text { at } z=0, \\
& M_{z}=\psi_{z z}=\psi=\theta_{z}=0 \text { at } z=1,
\end{aligned}
$$

where $\gamma=\frac{C H}{\nu_{V}}$ is the ratio of the spin-down time due to the drag to the viscous timescale; the bottom drag relaxes the angular momentum $M$ to the local planetary value $\Omega a c^{2}$ through a drag coefficient $C$.

The model flow started from an isothermal state at rest and is maintained by a Newton heating function where the heating rate is proportional to the difference between the model potential temperature and a specified radiativeconvective equilibrium temperature distribution, which follows the HH80 one:

$\theta_{\mathrm{E}}=\frac{4}{3}-y^{2}+\frac{\Delta_{\mathrm{V}}}{\Delta_{\mathrm{H}}}\left(z-\frac{1}{2}\right)$.

Equation (6) is used extensively in dry axisymmetric models (e.g., HH80, Farrell, 1990; Cessi, 1998) and it is related to the thermal forcing term of the equation system. A statically stable state as a vertical profile of $\theta_{\mathrm{E}}$ is also assumed 
by Eq. (6). HH80 suggested that the impact of latent heat released by water vapor condensation can be incorporated into dry axisymmetric models by modifying the meridional distribution of $\theta_{\mathrm{E}}$. HL92 followed the HH80 argument and altered the concentration of $\theta_{\mathrm{E}}$ under the constraint of equal energy input. The resulting $\theta_{\mathrm{E}}$ distributions used by HL92 were peaked distributions on and off the Equator, resulting in a stronger Hadley circulation with respect to the circulation obtained by applying Eq. (6). Tandon et al. (2013) used narrow and wide thermal forcing to mimic El Niño or global warming effects on a tropical circulation in a global circulation model. On the opposite side, in fact, we can suppose that, if a warmer climate happens, especially in the tropical regions, a very weak gradient of the equilibrium temperature $\theta_{\mathrm{E}}$ will have a greater extent in latitude, expanding consequently the tropical region. This has already occurred in the past, especially in the middle Cretaceous and Eocene, when the tropics extended up to $60^{\circ}$. This is the so-called equable climate (e.g., Greenwood and Wing, 1995) where roughly equal temperatures are present throughout the world. During those geological ages, the temperature was generally higher everywhere, but adding a constant to the temperature does not change the response of this kind of model. The Equatorpole temperature gradient was smaller than the present situation, whereas we prescribe a constant surface Equator-pole $\theta_{\mathrm{E}}$ gradient. As we shall show afterwards, this is necessary to demonstrate that it is the tropical temperature gradient that drives the Hadley circulation. Thus, in order to study systematically these different conditions, we adopt the strategy to build forcing functions dependent on a parameter that controls the $\theta_{\mathrm{E}}$ gradient in the tropical regions. Since, with different horizontal distributions of $\theta_{\mathrm{E}}$, we can figure out that, even the vertical distribution could be affected by some physical mechanisms that make the atmosphere more or less stable than the stratification described by the $z$ component of Eq. (6). The meridional and vertical changes of equilibrium temperature can be obtained by changing the exponents of $y$ and $z$ in Eq. (6), transforming Eq. (6) in the following equation:

$\theta_{\mathrm{E}}=\frac{4}{3}-|y|^{n}+\frac{\Delta_{\mathrm{V}}}{\Delta_{\mathrm{H}}}\left(z^{k}-\frac{1}{2}\right)$.

The terms $n$ and $k$ control the horizontal distribution of $\theta_{\mathrm{E}}$ and its stratification, respectively. Small values of $n$ are associated with concentrated $\theta_{\mathrm{E}}$ distributions. Increasing $n$ means increasing the broadness of the $\theta_{\mathrm{E}}$ distribution. Values of $k$ larger than 1 mean more stable stratification at upper levels; vice versa, smaller $k$ values mean that lower levels are more stable than upper levels. Thus, it becomes quite natural to explore the response of the Hadley circulation by changing the parameters $n$ and $k$, which control the distribution of $\theta_{\mathrm{E}}$, in the closest ranges of 2 and 1 , respectively. Thus, $n$ and $k$ will change from 0.5 to 3 with a 0.5 step, in such a way as to have a set of 36 simulations. When $n=2$ and $k=1$, Eq. (7) becomes Eq. (6), and the experiment performed with such
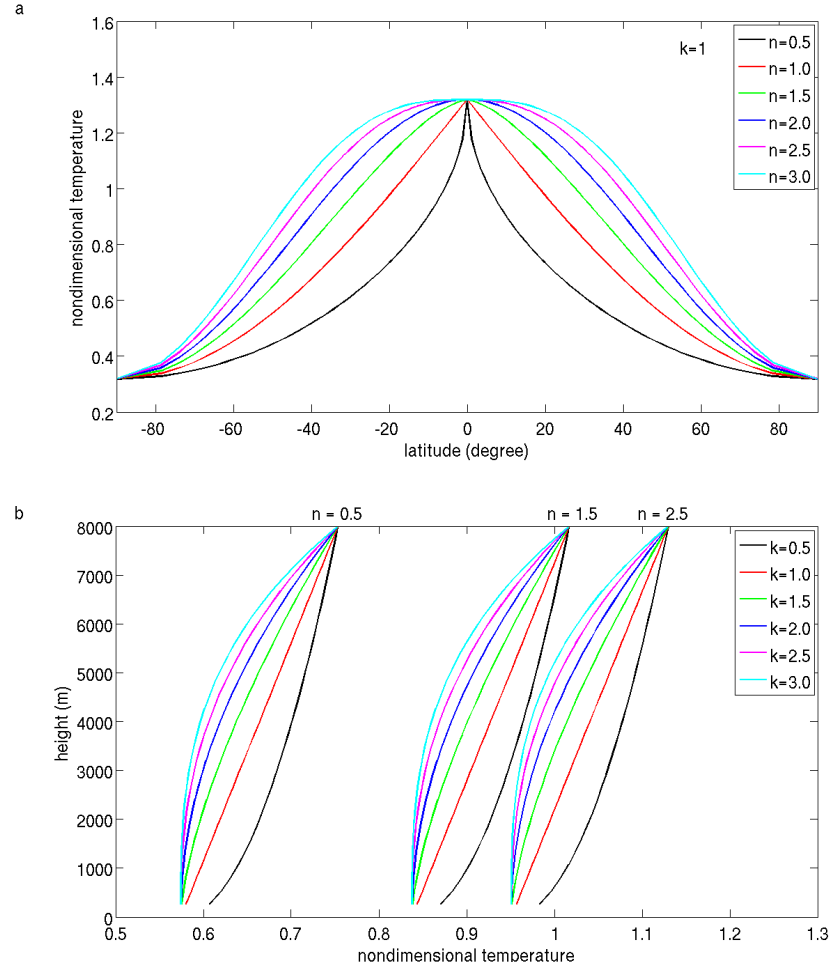

Figure 1. Vertical (a) and meridional (b) average of nondimensional equilibrium temperature as a function of $n$ with $k=1$ (a) and $k$ with $n=0.5,1$ and 1.5 (b). Dimensional values are obtained by multiplying by $\theta_{0}=300 \mathrm{~K}$.

values of $n$ and $k$ will be considered the reference experiment.

The meridional and vertical averages of $\theta_{\mathrm{E}}$ are shown in Fig. 1. Heating functions with an $n$ value equal to 0.5 should not be regarded as unreal, but merely as a simple way of representing a specific state of the atmosphere. The same assertion is valid for all other parameters. As $n$ increases, the average temperature increases as well, but the meridional gradient decreases in the tropical regions (Fig. 1a).

With the prescribed $\theta_{\mathrm{E}}$ as specified by Eq. (7), $\theta_{\mathrm{E}}$ values at the boundaries and its Equator-pole difference temperature remain invariant with respect to $n$, for a given $k$ value. The energy input is not constant here, which differs from HL92, which analyzed the influence of concentration heating perturbing the forcing function $\theta_{\mathrm{E}}(y, z)$ in such a way that $\theta_{\mathrm{E}}$ averaged over the domain remained constant. It is easily visible in Fig. 1b. Higher $n$ values, keeping $k$ invariant, have higher averaged $\theta_{\mathrm{E}}$ at all levels. The same is true for $k$, with higher $k$ values, for $n$ constant; $\theta_{\mathrm{E}}$ at each level is always higher than that with lower $k$ values. The Equator-pole $\theta_{\mathrm{E}}$ differences at upper and lower vertical boundaries are the same for all the experiments having the same $k$; the vertical averaged $\theta_{\mathrm{E}}$ changes as a function of $k$, for $n$ constant.

Whether global warming makes the equilibrium temperature distribution narrower or wider is beyond the aim of the 
paper. One can expect that global warming will broaden the temperature distribution, but, at the same time, it could have an impact above all on the sea surface temperature (SST), bringing more water in the upper atmosphere, which changes the vertical distribution, especially of the temperature in the intertropical convergence zone (ITCZ). It is supposed that, in first approximation, oceans force the atmosphere, so we have to allow for the possibility that increasing SST can change the forcing distribution. Increasing uniformly SST the Hadley cell might show a poleward expansion, as showed by Chen et al. (2013) by an aqua-planet model, but in that case, the mechanism was supposed to be related mainly to mid-latitude eddies rather than a tropical forcing. Since other causes can change the temperature distribution of a planet, such as changes in the solar activity for instance, we will focus on the temperature distribution regardless of its cause.

In this model, the atmosphere is dry, as in many other studies (e.g., Schneider, 1977, HH80, Caballero et al., 2008); changing the $\theta_{\mathrm{E}}$ distribution allows for a change in the static stability. Looking at the average $\theta_{\mathrm{E}}$ along the vertical direction, low values of $k$ are related to low values of static stability, especially at higher levels of the model atmosphere.

The Brunt-Väisälä frequency, when the atmosphere reaches the equilibrium, will be

$N^{2}=\frac{\left(g k \Delta_{\mathrm{V}} / \Delta_{\mathrm{H}} z^{(k-1)}\right)}{\left[4 / 3-y^{n}+\Delta_{\mathrm{V}} / \Delta_{\mathrm{H}}\left(z^{k}-1 / 2\right)\right]}$.

It is clear from Eq. (8) that the Brunt-Väisälä frequency does not depend on $n$ at the poles and Equator. On the contrary, it depends on $k$; large values of $k$ imply a more stable atmosphere in the upper levels, especially at the poles, making the model atmosphere more similar to the real one, simulating in some respects a sort of tropopause. Moreover, this is equivalent to creating a "physical" sponge layer in the upper levels of the model that will have some effects on the vertical location of the stream function maximum.

Starting from Eq. (7), a set of experiments were performed changing $n$ and $k$ in such a way as to have a set of numerical results. In order to isolate the contribution of the $\theta_{\mathrm{E}}$ distribution to the solution of Eq. (3), a set of parameters will be used:

$$
\begin{aligned}
& a=6.4 \times 10^{6} \mathrm{~m} \quad \Omega=2 \pi /\left(8.64 \times 10^{4}\right) \mathrm{s}^{-1} \\
& \Delta_{\mathrm{H}}=1 / 3 \quad \Delta_{\mathrm{V}}=1 / 8 \\
& g=9.8 \mathrm{~m} \mathrm{~s}^{-2} \quad C=0.005 \mathrm{~m} \mathrm{~s}^{-1} \\
& H=8 \times 10^{3} \mathrm{~m} \quad \tau=20 \text { days } \\
& v_{\mathrm{V}}=5 \mathrm{~m}^{2} \mathrm{~s}^{-1} \quad \nu_{\mathrm{H}}=1.86 \mathrm{~m}^{2} \mathrm{~s}^{-1}
\end{aligned}
$$

The parameters in Eq. (9) are the same as those used by Cessi (1998).
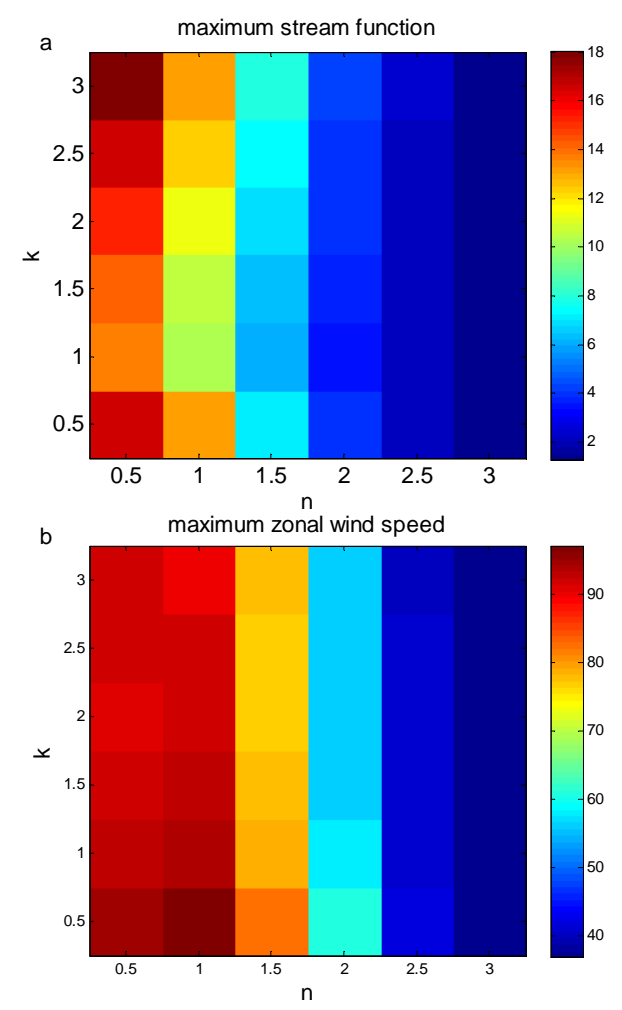

Figure 2. Maximum non-dimensional stream function (a) and zonal wind speed $\left(\mathrm{m} \mathrm{s}^{-1}\right)$ (b) as a function of parameters $n$ and $k$ for the steady solution. Dimensional values of the stream function are obtained by multiplying by $\nu_{\mathrm{V}} R \varepsilon^{-1}=484 \mathrm{~m}^{2} \mathrm{~s}^{-1}$.

\section{Numerical results}

This section is divided into three subsections, the first showing the results of the model applying the equinoctial condition, when the Sun is assumed to be over the Equator. The solution is steady, as already shown for instance in Cessi (1998). The second subsection will show the results of the model having a $\theta_{\mathrm{E}}$ distribution described by Eq. (7) but moving following a seasonal cycle. Finally, the case $n=2$ and $k=1$ will be discussed in the third subsection in comparison with previous studies.

\subsection{Equinoctial simulations}

The axially symmetric circulation is forced by axially symmetric heating as in HH80 and many others and as prescribed by Eq. (7). The model started from an isothermal state and was run for 300 days, even though it reached its equilibrium approximately after 100 days, in order to be sure that the model does not have instabilities in the long run. The stream function values obtained when $n=2$ and $k=1$, i.e., the reference experiment, are about the same as those obtained by HH80. We will show the non-dimensional value, but to have the dimensional values, we need to multiply by $\nu_{\mathrm{V}} R \varepsilon^{-1}=484 \mathrm{~m}^{2} \mathrm{~s}^{-1}$. 
The absolute value of the maximum stream function intensity under the equilibrium conditions for the 36 experiments is shown in Fig. 2. When $n=0.5$, with $k$ constant, the circulation is always the strongest. The stream function intensity is inversely proportional to $n$ (Fig. 2a). With $n=0.5$, the experiment resembles the one described in HL92, where they concentrated the latitudinal extent of heating, and this led to a more intense circulation. However, they imposed the forcing function $\theta_{\mathrm{E}}(x, y)$ in such a way that its average over the domain remained the same as in the control experiment, i.e., without changing the energy input. They found that concentration of the heating through a redistribution of heat within the Hadley cell led to a more intense circulation without altering its meridional extent. Instead, here, it is evident from Fig. 1 that the experiment with $n=0.5$ has an energy input lower than the other cases. Nevertheless, the Hadley circulation is always more intense than the other cases and opposite to higher $n$ value experiment results; the circulation is confined closer to the Equator. Thus, the results of HL92 are extended to a more general case with a lower energy input. It is worth noticing that the constraint of an equal Equator-pole gradient of mean $\theta_{\mathrm{E}}$ is assumed here differently from HL92 (Fig. 1a).

The dependence on $k$ is not as straightforward as the one on $n$, instead. The stream function reaches the highest value for $n=0.5$ and $k=3$. With high $n$ values, the Hadley cell strength is lower, and the dependence on $k$ loses its importance. In other words, in our model, the symmetric circulation strength is modulated by $k$ only when the equilibrium temperature distribution is concentrated on the Equator.

Figure $2 \mathrm{~b}$ shows the maximum zonal wind speed as a function of $n$ and $k$, it is inversely proportional to $n$, the dependence on $k$ is not as clear as the one on $n$ and, when $n=3$, it almost vanishes in accordance with the behavior of the maximum stream function. These results are in agreement with HL92, who found a stronger zonal wind when the forcing was concentrated at the Equator.

Some observational studies define the border of the Hadley cell where the stream function goes to 0 at $500 \mathrm{hPa}$ (e.g., Frierson et al., 2007). Since, in our model, the zero stream function is at the poles, it is problematic to define an edge of the Hadley cell based on the zero stream function. Moreover, circulation intensity changes greatly in our experiments, so it is puzzling to define an edge of the Hadley cell based on an absolute value of the circulation itself. Hence, we will look at the location of the maximum stream function, and we will analyze its poleward shift as a function of the two parameters $n$ and $k$. The edge of the cell might also be defined by values of isolines that are relative with respect to the maximum value, for example $1 / 4$ of the stream function. For the sake of clarity, this definition would be an operational one, and does not follow the definition used in observational studies, for example by Dima and Wallace (2003) or Frierson et al. (2007).
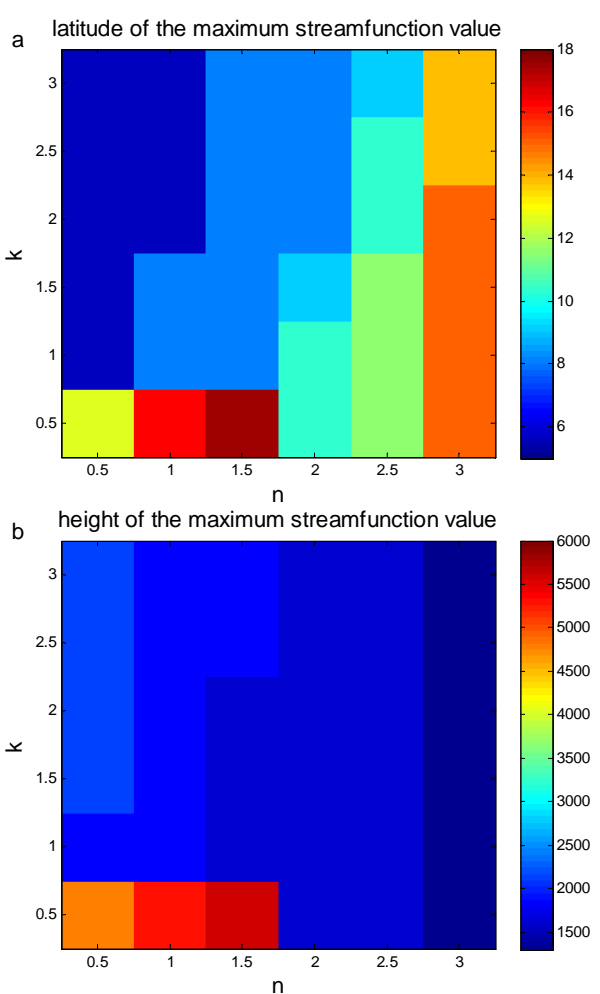

Figure 3. Latitude (degrees) (a) and height (m) (b) of the maximum non-dimensional stream function as a function of parameters $n$ and $k$.

The latitude of the maximum stream function value shows a general dependence on $n$ and $k$. It increases with $n$ and decreases with $k$. However, as shown in Fig. 3a, this dependence is not straightforward or linear, although we have a few exceptions, for instance when $k=n=0.5$. Hence, in general, when $n$ increases, and the temperature gradient in the tropics decreases, even though the total energy input is larger, the stream function is weaker and the Hadley cell moves poleward. This result is in agreement with other model outcomes (Frierson et al., 2007; Lu et al., 2008; Gastineau et al., 2008; Tandon et al., 2013). The model predicts a weakening of circulation, in contrast to the strengthening, together with widening of the Hadley circulation for the past three decades observed by Liu et al. (2012) and $\mathrm{Hu}$ and Fu (2007). However, Liu et al. (2012) showed that if the observations start from 1870, the Hadley cell has become more narrow and stronger.

The height of the maximum stream function value is confined for almost all the simulations to under $2200 \mathrm{~m}$ and the general rule is that, when $n$ increases, the height of the maximum decreases; however, a few experiments, those with $k=0.5$ and $n=0.5,1$ and 1.5 , have the maximum value between 4300 and $5600 \mathrm{~m}$ (Fig. 3b).

In general, the location of the maximum zonal wind speed does not show any evident relationship with the parameters $n$ 
Table 1. Latitudes (in degrees) of the maximum wind speed for the equinoctial and time-dependent solutions when $k=1$ as a function of the parameter $n$.

\begin{tabular}{lrlrlrl}
\hline$n$ & 0.5 & 1 & 1.5 & 2 & 2.5 & 3 \\
Equinoctial & 27.4 & 28.7 & 27.4 & 26.1 & 28.7 & 47.7 \\
Time dependent & 28.7 & 28.7 & 28.7 & 27.4 & 27.4 & 44.4 \\
\hline
\end{tabular}

and $k$. It is always confined between 26 and $29^{\circ}$ off the Equator; however, when $n=3$, there is an abrupt transition to about $48^{\circ}$, independently of the $k$ value. In Table 1 , we show the latitude of the maximum wind speed when $k=1$ for different $n$ values.

The difference between $\theta_{\mathrm{E}}$ and $\theta$, once the model reaches the equilibrium, is quite interesting when $k$ is not equal to 1. Figure 4 shows meridional distributions of $\theta_{\mathrm{E}}$ and $\theta$ for $n=3$ and $k=0.5,1$ and 3. In Fig. $4 \mathrm{a}, k=0.5$, and $\theta_{\mathrm{E}}$ is under $\theta$; when $k=1$, we find that $\theta_{\mathrm{E}}$ is over $\theta$ in a region around the Equator (Fig. 4b), with $\theta_{\mathrm{E}} \operatorname{crossing} \theta$ at about $47^{\circ}$, finding again the equal area condition suggested by HH80, and that explains approximately the jet location, whereas in Fig. $4 \mathrm{c}$, with $k=3$, we can see how $\theta_{\mathrm{E}}$ is over $\theta$. Nevertheless, all simulations with $n=3$ give almost the same solution, in terms of circulation strength and jet location (Figs. 2 and 3). For other values of $n$, the results are similar, but the differences between $\theta_{\mathrm{E}}$ and $\theta$ are not so visible.

We can understand these findings in the light of Cessi (1998), who analyzed the model described by the set of Eq. (3) by using an asymptotic expansion of the variables $M, \theta$ and $\psi$ in power series of the Rossby number $R$. The meridional advection, in the nonlinear term of the expansion, depends on the differences between $\theta_{\mathrm{E}}$ and $\theta, \theta_{\mathrm{E}}-\theta$; on the cube of the meridional temperature gradient; and quasilinearly on the imposed stratification. Deducing that for unstable stratification, this term would appear as a negative diffusivity term, a condition that can exist even with some stable stratifications (Cessi, 1998). This seems to be our case when $k=0.5$. Although the stratification imposed by Eq. (7) is stable, i.e., $\frac{\partial \theta_{\mathrm{E}}}{\partial z}>0$, the second derivative is negative when $k=0.5$, reducing the stability at upper levels, so this situation can be seen as a way of simulating the effect of the latent heat released by water vapor condensation. Running the model with an enhanced vertical viscosity (5 times the value defined in Eq. 9), the situation described by Fig. 4a changes to look like that of Fig. 4b. Defining stratification with $k=0.5$ is consequently equivalent to reducing the actual vertical diffusivity.

When $k=3$, the air in upper levels is very stable, and the upward flow has to do more work to rise at upper levels; most of the thermal energy that drives the model atmosphere is evidently dissipated by this work, reducing the actual energy with respect to that provided by $\theta_{\mathrm{E}}$. We performed some runs with reduced vertical viscosity; the actual value of $\theta$ in Fig. $4 \mathrm{c}$ slightly increases, becoming closer to $\theta_{\mathrm{E}}$, but it re-
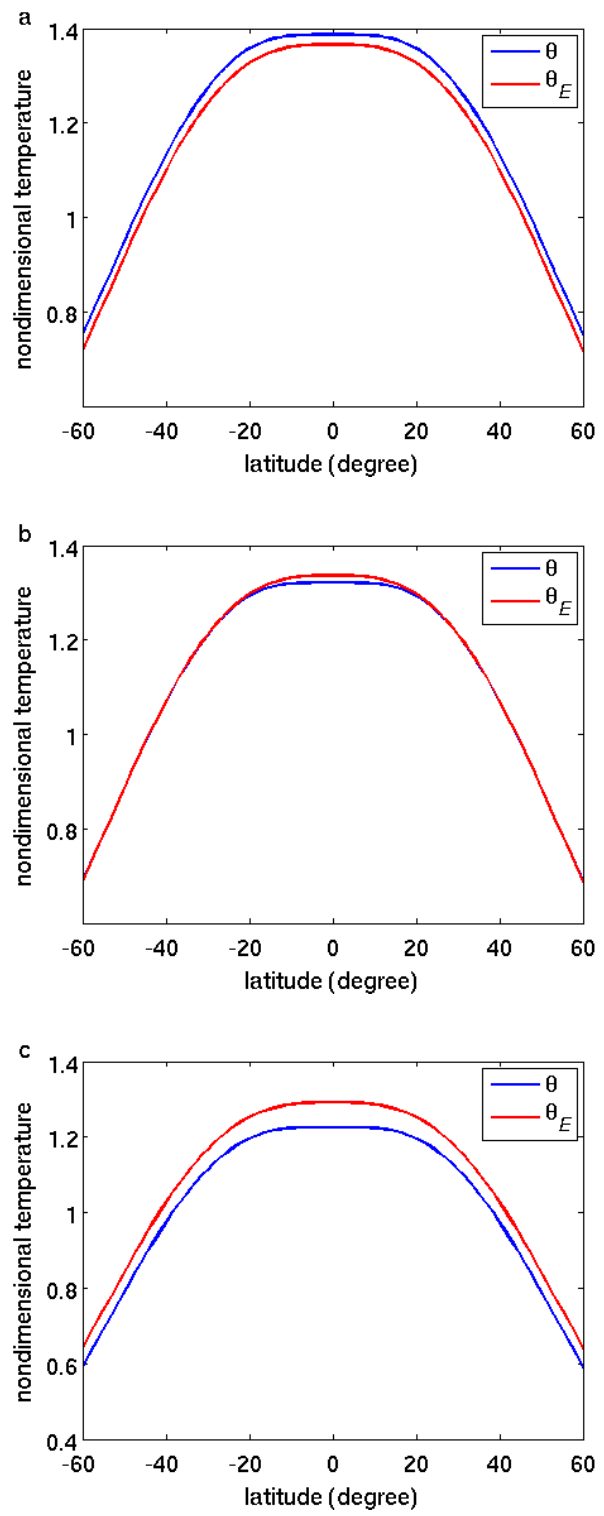

Figure 4. Vertically averaged $\theta$ (blue line) and $\theta_{\mathrm{E}}$ (red line) for the simulations with $n=3$ and $k=0.5$ (a), $k=1$ (b) and $k=3$ (c). Dimensional values are obtained by multiplying by $\theta_{0}=300 \mathrm{~K}$.

mains constant under the $\theta_{\mathrm{E}}$ curve, even for values of vertical viscosity close to 0.1 (with vertical viscosity very close to 0 or negative, the model blows up). This should not be interpreted as an unphysical result, but it has to be seen as the difficulty of flow temperature in relaxing to $\theta_{\mathrm{E}}$ because of very stable imposed stratification. In any case, the Hadley circulation is still reproduced, demonstrating the robustness of the model.

With $n$ getting larger, the $\theta_{\mathrm{E}}$ distribution becomes flatter in the tropical region and $\theta$ clamps to $\theta_{\mathrm{E}}$. In general, we expect that a vigorous circulation will occur in a fast rotating planet unless the thermal gradient becomes small in the tropics. In such a case, the angular momentum homogenization 
is equivalent to a weakening of the rotation (Cessi, 1998). If the circulation is proportional to the cube of the meridional temperature gradient, it is quite evident that, when such a gradient has high values in the tropical region, the circulation is vigorously driven by this term, whereas, when it approaches zero, it is the term $\theta_{\mathrm{E}}-\theta$ that dominates. HH80 found that the edge of the Hadley cell was at the mid-latitudes when the planetary rotation was lower than that of the Earth. Since this phenomenon is observed here for a wider forcing distribution, this common result may be attributed to the process of homogenization of momentum and temperature in the equatorial region.

In order to explain equable climates like those supposed to have occurred in the Cretaceous and Eocene, Farrell (1990) formulated an axisymmetric model starting from that of Held and Hou and used a forcing with $n=2$ and $k=1$, introducing a radiative-diffusive term to make flatter the model temperature gradients in the tropics. For high values of $n$, the $\theta$ distributions are similar to those obtained by Farrell (1990), with high values of its diffusive parameter. In some respects, flattening of the forcing distribution is equivalent to having a diffusive term, and this also explains Fig. 4c. The Farrell (1990) model showed a poleward shift of the zonal jet, and it has to be noticed that a poleward shift of the subtropical jets was also observed by HH80 when increasing the vertical viscosity.

Figure 5 shows the stream function and the zonal wind speed for the experiments $n=k=0.5$ (Fig. 5a) and $n=k=3$ (Fig. 5b). The parameter $n$ controls the Hadley cell and jet stream widths. The results show that, with $n=k=0.5$, the Hadley cell and jet streams are quite narrow. As far as the vertical position of the maximum value of the stream function is concerned, experiments with $k=0.5,1$ and 1.5 exhibit particular behavior with respect to the other experiments. The stream function has its maximum at upper levels. This is related to the different stratification imposed by the parameter $k$. Stratification with low values of $k$ favor air to move to higher levels with respect to experiments with higher $k$ values.

\subsection{Time-dependent simulations}

Since heating depends on solar irradiation, it is of interest to analyze the solutions obtained by the annually periodic thermal forcing and to compare it with the steady solutions described previously in this paper. Starting from Eq. (7), we can formulate an equilibrium temperature distribution having the maximum heating off the Equator at latitude $y_{0}$ :

$\theta_{\mathrm{E}}=\frac{4}{3}-\left|y-y_{0}\right|^{n}+\frac{\Delta_{\mathrm{V}}}{\Delta_{\mathrm{H}}}\left(z^{k}-\frac{1}{2}\right)$,

where $y_{0}$ in Eq. (10) is dependent on time according to

$y_{0}(t)=\sin \left(\frac{\varphi_{0} \pi}{180}\right) \cdot \sin \left(\frac{2 \pi t}{360 \text { days }}\right)$, a
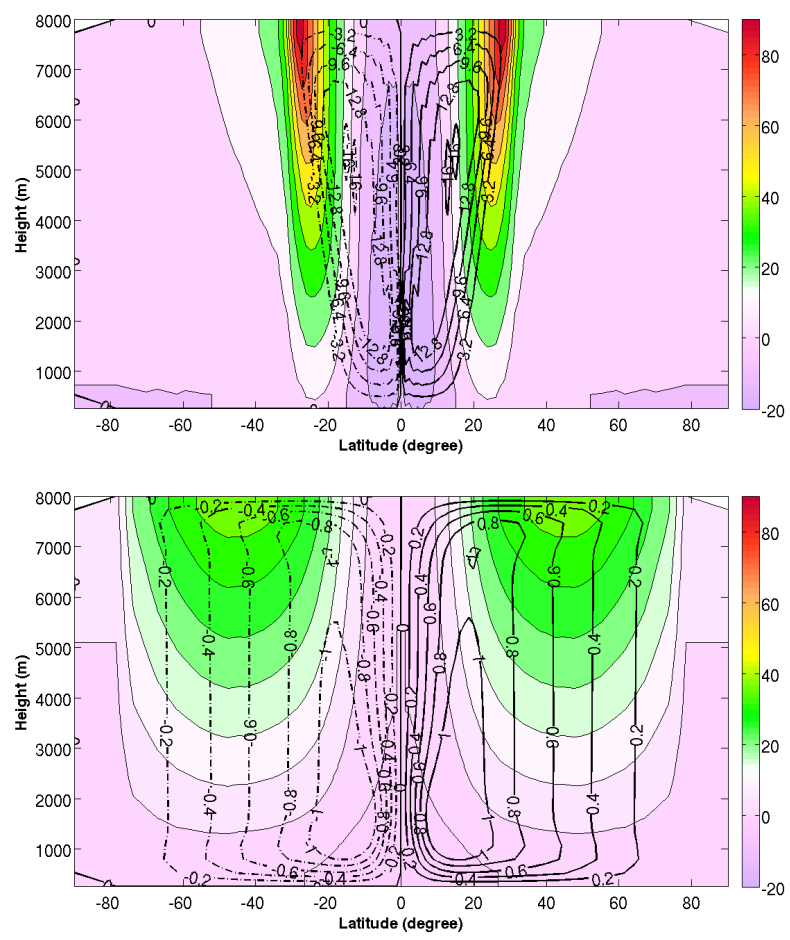

Figure 5. Non-dimensional stream function (contours) and zonal wind speed $\left(\mathrm{m} \mathrm{s}^{-1}\right)$ (colors) for the steady cases $n=0.5, k=0.5$ (a) and $n=3, k=3$ (b). Dimensional values of the stream function are obtained by multiplying by $\nu_{\mathrm{V}} R \varepsilon^{-1}=484 \mathrm{~m}^{2} \mathrm{~s}^{-1}$.

where $\varphi_{0}$ is the maximum latitude off the Equator where heating is maximum. Equation (10), with $n=2$ and $k=1$, and Eq. (11) are the same used by Fang and Tung (1999), with the choice of the maximum extension of $\varphi_{0}$ consistent with the choice of Lindzen and Hou (1988); i.e., $\varphi_{0}=6^{\circ}$. A prescribed equilibrium temperature varying seasonally makes the simulations more realistic. We will focus on the average and maximum values, in absolute terms, of the stream function and zonal speed obtained during 360 days of simulations. The averaged values are obtained in these cases by averaging the outputs obtained every 30 days, starting from the minimum corresponding to the summer Hadley cell in the boreal hemisphere.

The annual averages of the time-dependent and equinoctial circulations show that maximum stream functions and zonal wind speeds behave quite similarly (Fig. 6); nevertheless, the instantaneous Hadley circulation almost never resembles the modeled circulation (Fang and Tung, 1999) or the real one (Dima and Wallace, 2003).

The maximum stream function is obtained here when $k=n=0.5$ (Fig. 6a). In general, for $n=0.5$, we have stronger circulations and winds. These simulations confirm the inverse relationship between stream function strength and $n$. The circulation strength expressed as an annually averaged value is weaker when compared with that obtained in 

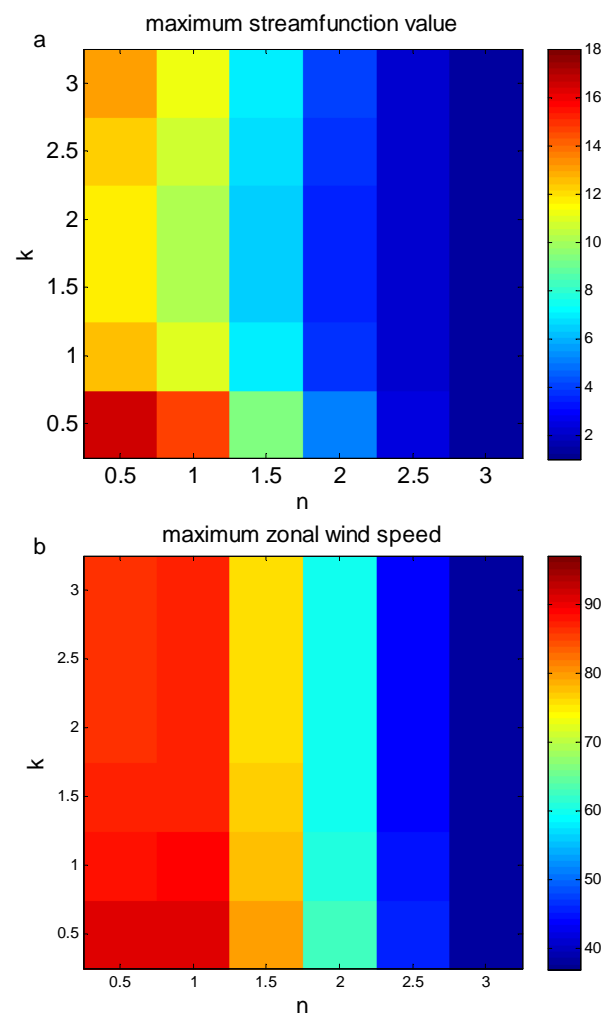

Figure 6. Maximum of the annually averaged non-dimensional stream function (a) and zonal wind speed $\left(\mathrm{m} \mathrm{s}^{-1}\right)(\mathbf{b})$ as a function of parameters $n$ and $k$ for the time-dependent simulations. Dimensional values of the stream function are obtained by multiplying by $v_{\mathrm{V}} R \varepsilon^{-1}=484 \mathrm{~m}^{2} \mathrm{~s}^{-1}$.

the equinoctial experiments, when $n$ is low and $k$ is high; otherwise, it is only slightly stronger, but it is never twice as strong as that of the equinoctial solution as found by Fang and Tung (1999). When $n=2$ and $k=1$, our results are consistent with those obtained by Walker and Schneider (2005) as discussed in Sect. 3.3. There is no analog maximum when $n=0.5$ and $k=3$ found in the steady solution. The maximum of the annually averaged wind speed shows only a slight dependence on $k$ when $k$ is low.

The meridional position and the height of the maximum stream function show that there is no clear dependency on $n$ and $k$ (Fig. 7). The difference between the time-dependent simulations and the steady solutions is quite interesting. It is to be noticed that the latitude of the stream function maximum in the time-dependent solution is in the range from 12.5 to $16^{\circ}$ (Fig. 7a), whereas, in the equinoctial solutions, the corresponding latitude is within a larger range. The maximum stream function is located at higher levels, between 4500 and $6000 \mathrm{~m}$, when $k$ is equal to or less than 1 and when $n$ is less than 2.5. Otherwise, the maximum is positioned under $3000 \mathrm{~m}$ (Fig. 7b). The location and strength of averaged results are impressively similar to those obtained by experiments with steady solutions.
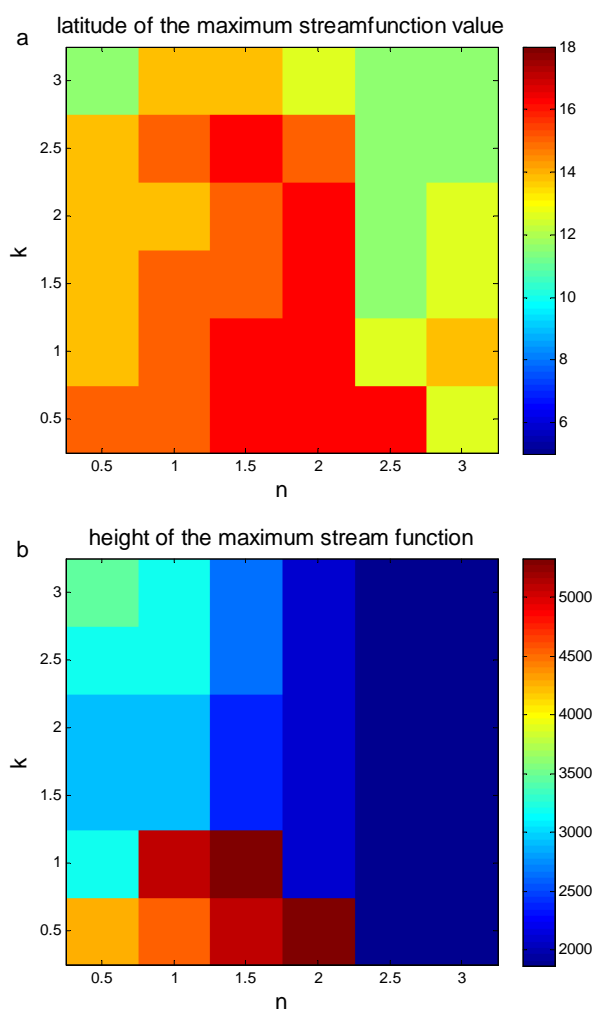

Figure 7. Latitude (degrees) (a) and height (m) (b) of the maximum annually averaged non-dimensional stream function for the timedependent solution.

More than the steady solution, it is evident that the height of the maximum stream function is lower when $k=3$. In the steady solution, this phenomenon is not that evident. When $k=3$, the vertical gradient of $\theta_{\mathrm{E}}$ is higher at upper levels, making those levels more stable, and it prevents, evidently more than the equinoctial solution, air from moving higher, leaving circulation to occur at lower levels. The case $k=3$ is equivalent to imposing a "natural" sponge layer at the top of the model. Thus, it does not come as a surprise that the maximum stream function is lower than those observed in simulations with other $k$ values. This result is analogous to that of Walker and Schneider (2005) that removed the maximum stream function at higher levels found by Lindzen and Hou (1988), adopting a numerical sponge layer at the top of the model. A comparison with previous works of the simulations with $n=2$ and $k=1$ will be discussed in Sect. 3.3. On the contrary, with low $k$ values, the presence of a weaker $\theta_{\mathrm{E}}$ gradient at upper levels favors air to move higher, and the maximum stream function is observed at upper levels.

The position of the jet stream is almost similar to the one observed in the steady solution. It is confined between 28 and $30^{\circ}$, with the latitude of the averaged jet remaining almost in the same place, except when $n=3$; the jets are located at about $44^{\circ}$, confirming the abrupt transition of the jet stream position when $n=3$ already found for the equinoctial exper- 

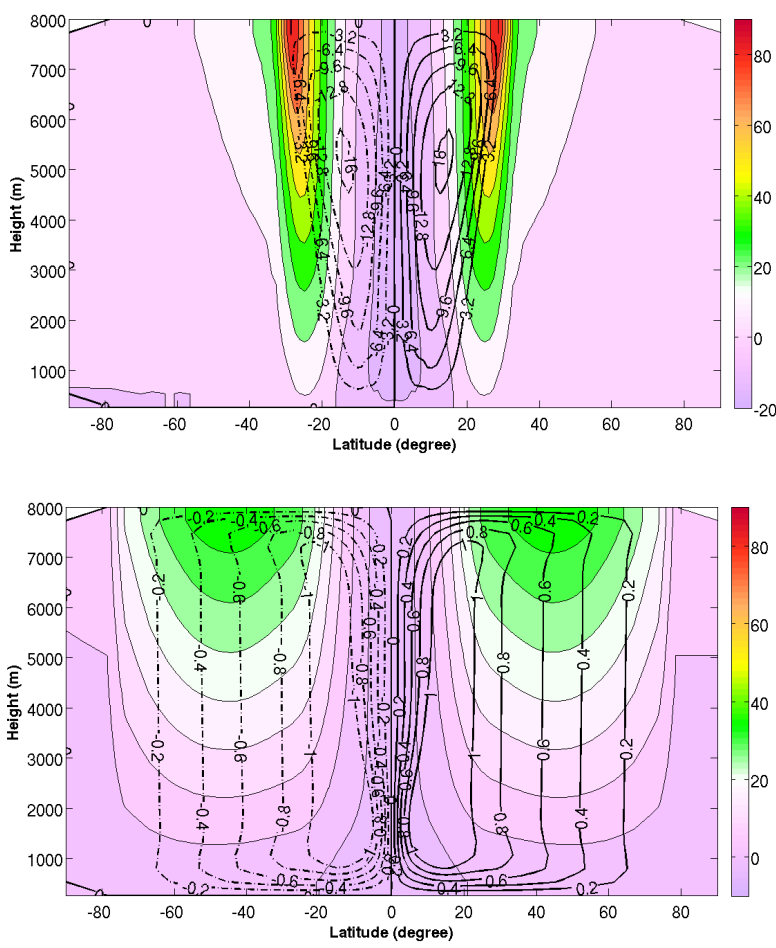

Figure 8. Annually averaged non-dimensional stream function (contours) and zonal wind speed $\left(\mathrm{m} \mathrm{s}^{-1}\right)$ (colors) for the steady cases $n=0.5, k=0.5$ (a) and $n=3, k=3$ (b). Dimensional values of the stream function are obtained by multiplying by $\nu_{\mathrm{V}} R \varepsilon^{-1}=484 \mathrm{~m}^{2} \mathrm{~s}^{-1}$.

iment. Fu and Lin (2011) suggest that the jets have moved poleward at a rate of about $1^{\circ}$ per decade in the last several years, but Strong and Davis (2007) observed that the Northern Hemisphere subtropical jet shifted poleward over the eastern Pacific, while an equatorward shift of the subtropical jet was found over the Atlantic basin. Excluding the case $n=3$, all the other subtropical jets in the different experiments have the position of the maximum very close to one another, and the shifting range is very limited. Thus, when a vigorous circulation occurs, the jet location must be located at about $30^{\circ}$, whereas, reducing the tropical gradient too much, the homogenization looks like that of a slowly rotating planet, and this is confirmed in the time-dependent solution. Both Tandon et al. (2013) and Kang and Polvani (2011) found a discrepancy in this area with the jets that do not follow the Hadley cell edge. In an axisymmetric model, defining the Hadley edge as a function of the stream function and connecting it to the jet location is problematic because of the lack of a zero value of the stream function.

Figure 8 shows the annually averaged circulation for the same cases as shown in Fig. 5, which is obtained by annually averaged heating. It is impressive how the steady and time-dependent solutions resemble each other. As in Fang and Tung (1999), the annual mean meridional circulation has
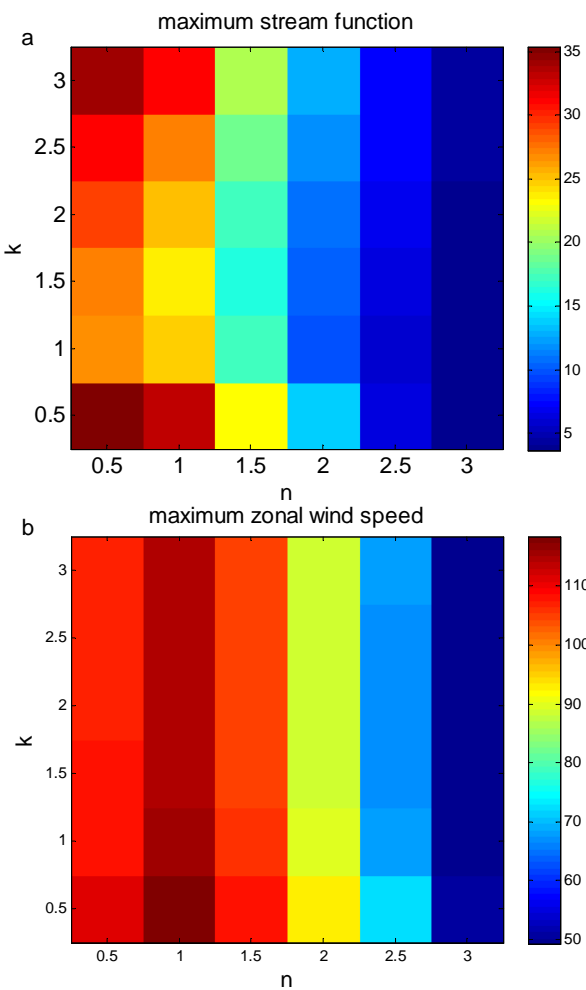

Figure 9. Maximum of the non-dimensional stream function (a) and zonal wind speed $\left(\mathrm{ms}^{-1}\right)(\mathbf{b})$ as a function of parameters $n$ and $k$ for the time-dependent simulations. Dimensional values of the stream function are obtained by multiplying by $\nu_{\mathrm{V}} R \varepsilon^{-1}=484 \mathrm{~m}^{2} \mathrm{~s}^{-1}$.

the same extent, but different from them, the strength of the annual mean circulation of the time-dependent solution is almost the same as the steady solution.

When the heating center is off the Equator, the intensity of the winter cell is stronger, whereas the cell of the summer hemisphere is weak and sometimes almost absent. Figure 9 shows the maxima of the stream function and zonal wind speed at the winter solstitial as a function of $n$ and $k$. The maximum stream function as a function of $n$ and $k$ has the same configuration of the steady solution. Here, as expected, the maximum intensity of the meridional circulation (Fig. 9a) reached during the simulation is twice as strong as that of the steady solution or the annually averaged timedependent solution, and it has about the same strength of the observed circulation. However, the winds are much stronger too, in contrast to observations. The zonal wind has a different configuration instead; the maximum zonal wind speed is obtained when $n=1$ (Fig. 9b).

We can inspect a couple of simulations when the stream function reaches its maximum in the boreal hemisphere. Figure 10 shows the stream function and the zonal wind speed when $n=2$ and $k=0.5$ (Fig. 10a and b) and $n=2$ and $k=3$ (Fig. 10c and d). When $k=0.5$ (upper panels), the boreal (winter) circulation is much stronger when $k=0.5$, with the 

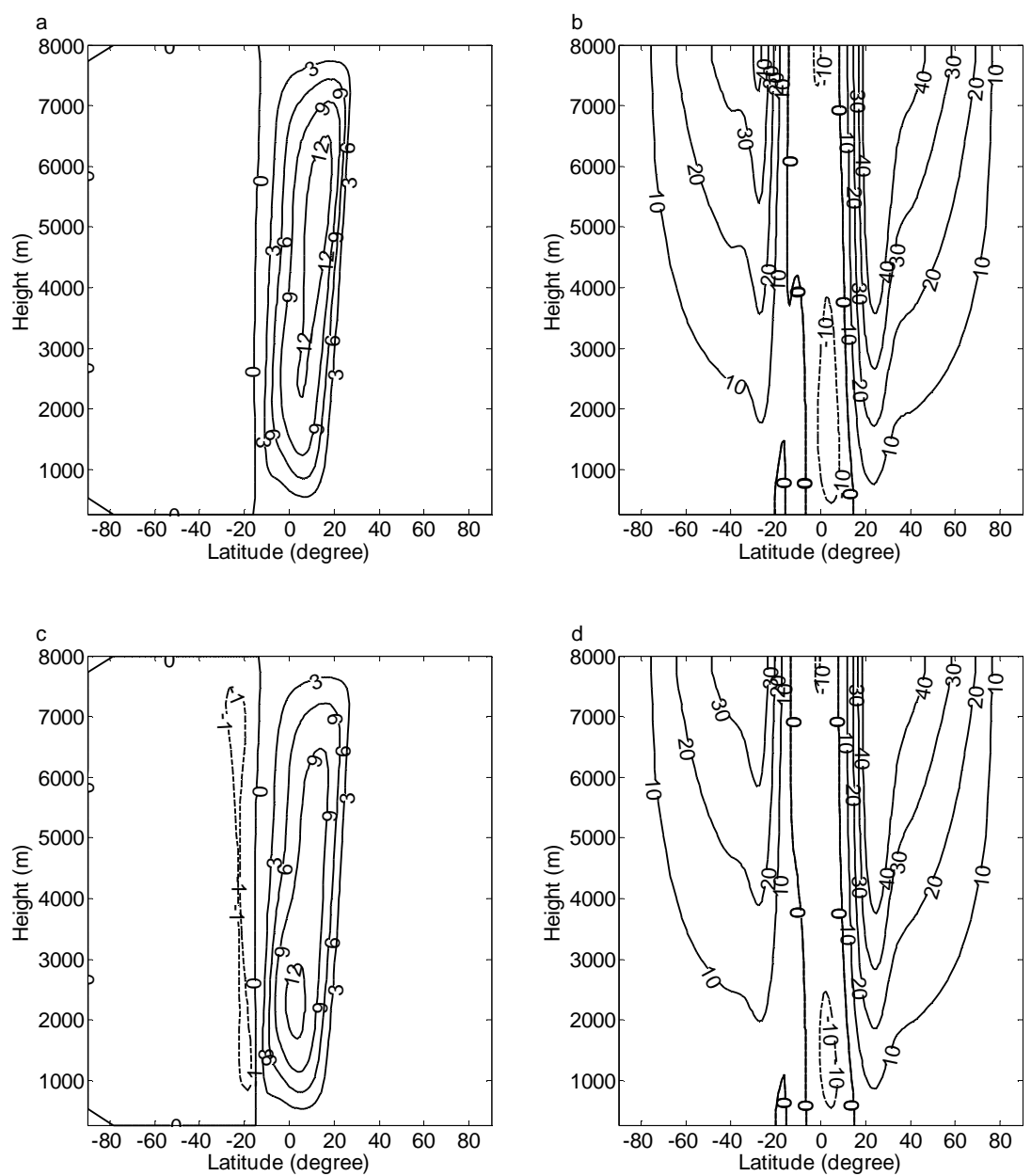

Figure 10. Boreal winter circulation, non-dimensional stream function (a, c) and zonal wind speed $\left(\mathrm{m} \mathrm{s}^{-1}\right)(\mathbf{b}, \mathbf{d})$ for the time-dependent simulation with $n=2, k=0.5$ (upper panels) and $n=2, k=3$ (lower panels). Dashed lines indicate negative values. Dimensional values of the stream function are obtained by multiplying by $\nu_{\mathrm{V}} R \varepsilon^{-1}=484 \mathrm{~m}^{2} \mathrm{~s}^{-1}$.

austral (summer) circulation almost absent. The vertical extent is larger and the maximum is located at higher levels. The summer and winter jets are both more intense than their counterparts for $k=3$. The tropical easterly winds are in this case stronger than those for $k=3\left(13.8 \mathrm{~m} \mathrm{~s}^{-1}\right.$ vs. $\left.11.4 \mathrm{~m} \mathrm{~s}^{-1}\right)$ and the easterly region is also wider. When $k=3$, it is noted that the winter cell is located closer to the Equator than the summer cell.

\subsection{A discussion on the case $n=2 k=1$}

When $n=2$ and $k=1$, corresponding to the classic case discussed in many studies, we found that the time-dependent solution is only slightly stronger than the steady solution. Lindzen and Hou (1988) proposed a study of the Hadley circulation in which the maximum heating was $6^{\circ}$ off the Equator. In their non-time-dependent model, the solution showed an average circulation much stronger with respect to the equinoctial solution. Lindzen and Hou (1988) suggested that this exceptional strength was due to a nonlinear amplifica- tion of the annually averaged response to seasonally varying heating, although Dima and Wallace (2003), in a study on the seasonality of the Hadley circulation, did not observe any nonlinear amplification.

With the parameters used for equinoctial and timedependent simulations, we performed an experiment like that of Lindzen and Hou (1988), with $\phi_{0}=6^{\circ}$, which will be referred to as the solstitial experiment. We found that the winter circulation is stronger by a factor of 3 with respect to the steady solution obtained with the equinoctial heating, consistent with the result of Walker and Schneider (2005). However, the average circulation obtained by averaging two solstitial experiments, with $\phi_{0}=6^{\circ}$ and $\phi_{0}=-6^{\circ}$, respectively, is only 1.5 times stronger than the steady solution with $\phi_{0}=0^{\circ}$, and it has a maximum at the upper levels of the model domain as in Lindzen and Hou (1988). We suggest that this maximum is due to a numerical effect caused by averaging the single solstitial experiments rather than a spurious effect caused by the rigid lid as suggested in Walker 
a
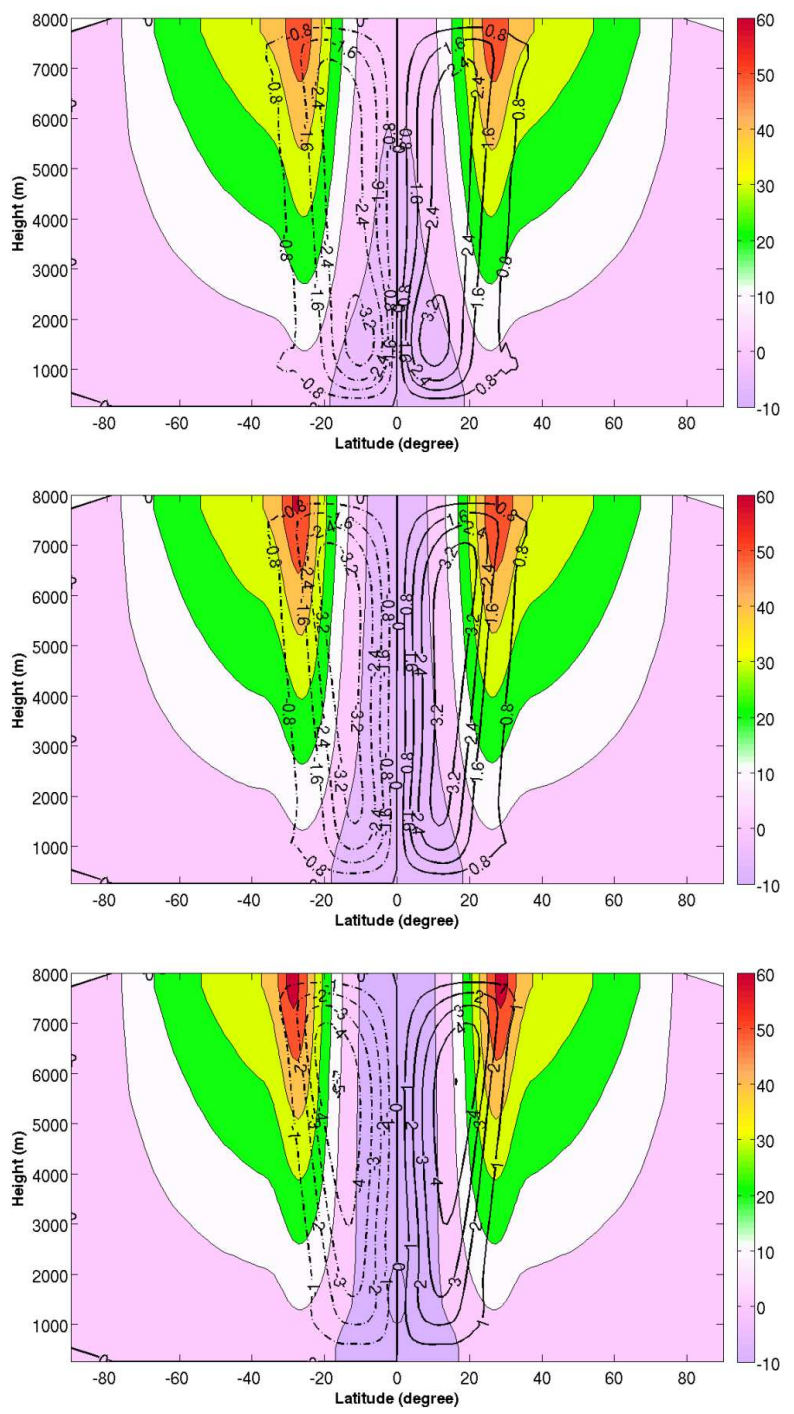

Figure 11. Non-dimensional stream function (contours) and zonal wind speed ( $\mathrm{m} \mathrm{s}^{-1}$; colors) when $n=2$ and $k=1$ for the steady solution (a), annually averaged for the time-dependent solution (b) and averaged for maximum heating $6^{\circ}$ off the Equator (c). Dimensional values of the stream function are obtained by multiplying by $v_{\mathrm{V}} R \varepsilon^{-1}=484 \mathrm{~m}^{2} \mathrm{~s}^{-1}$.

and Schneider (2005), even though a sponge layer actually lowers the maximum stream function height, and we can see the effects of a strong vertical gradient in the upper levels, especially in the time-dependent solution (cf. Figs. 3 and 7). Single solstitial experiments did not show a maximum in upper levels, as well as the equinoctial and time-dependent experiments (Fig. 11a and b). Consequently, the only operation performed to produce Fig. 11c, which exhibits the upperlevel maxima, was to average the two solstitial experiments, which causes the maximum at upper levels.

Finally, we notice that comparing the time-dependent solution with $\varphi_{0}=6^{\circ}$ with the equivalent steady solution hav- ing the heating off the Equator is not properly correct, since, for the time-dependent model, $\varphi_{0}$ represents only the maximum extension of heating; hence, a more correct comparison between steady and time-dependent solutions should be performed, with the time-dependent solution having $\varphi_{0}=3^{\circ}$. In such a case, the average solution is only slightly weaker than the Hadley circulation driven by annually averaged heating or by time-dependent heating, which does not show any maximum in the upper levels. Thus, the results of equinoctial, time-dependent and solstitial $\left(\varphi_{0}=3^{\circ}\right)$ experiments are mutually consistent.

\section{Conclusions}

The forcing of an Earth-like planet can change for several reasons. For instance, a change in forcing distribution can be caused by different factors such as global warming or longterm variation of solar activity.

Under the assumption of an equal Equator-pole difference at the surface, we used an axisymmetric model to study the sensitivity of the tropical atmosphere to different $\theta_{\mathrm{E}}$ distributions modulated by two parameters, $n$ that controls the broadness of the distribution and $k$ that modulates how the $\theta_{\mathrm{E}}$ is distributed vertically. Equinoctial and time-dependent solutions were simulated and compared. Moreover, for the case $n=2$ and $k=1$, corresponding to the classical distribution used in the literature, a few solstitial experiments were also run. When $n=2$ and $k=1$, the annually averaged circulations of equinoctial, time-dependent and solstitial experiments are quite close to one another, consistent with the results of Walker and Schneider (2005). However, the results differ from those of Lindzen and Hou (1988) and Fang and Tung (1999). As in all those works, the maximum of the stream function of the solstitial experiment is at upper levels, but it seems to be related to a spurious effect of the averaging operation rather than a spurious effect due to the rigid lid.

The results provide evidence that concentrated equilibrium temperature distributions enhance the meridional circulation and jet wind speed intensities, confirming findings of Lindzen and Hou (1988), even though these authors imposed the same energy input. However, in the present study, the concentrated distribution at the Equator has lower energy input.

The width of the Hadley cell is proportional to $n$, but when the cell width increases, its intensity decreases. A poleward shift of the Hadley circulation with warming is very robust, as has been observed in many models and over a large range of climates (Frierson et al., 2007). Since the Equator-pole gradient is the same for all the experiments with the same $k$, it is evident that the gradient in the tropical region controls the circulation strength. The term $k$ controlling the imposed stratification has an influence on the actual temperature distribution that can differ remarkably from $\theta_{\mathrm{E}}$ distribution. 
Vertical stratification is important in determining the position and intensity of the Hadley cell and jet when $n$ is low, whereas $k$ loses its importance when the $\theta_{\mathrm{E}}$ distribution is wider. This latter result is consistent with the results of Tandon et al. (2013), who found that the Hadley cell expansion and jet shift had relatively little sensitivity to the change in the lapse rate. Consequently, the subtropical jet stream intensities are controlled by the broadness of horizontal equilibrium temperature rather than by the stratification, with higher values of the jet when the thermal forcing is concentrated on the Equator. In the case of a time-dependent solution with $n=0.5$ (concentrated heating) and $k$ taking the extreme values ( 0.5 and 3$)$, the simulated maximum stream function has the same magnitude order as the observed stream function, 10 times larger than that obtained in HH80 and with the reference simulation, even though with stronger winds too.

The jet stream position does not show any dependence with $n$ and $k$, except when the $\theta_{\mathrm{E}}$ distribution is the widest $(n=3)$; in such a case, an abrupt change occurs and the maximum of the zonal wind jet is located at mid-latitudes ( $47^{\circ}$ in steady solution and $44^{\circ}$ in an annually averaged timedependent solution). This behavior can be explained by using the analytic study of this model performed by Cessi (1998), claiming that, when the meridional gradient becomes too small, the circulation behaves as that of a slow rotating planet that exhibits a poleward shift of the subtropical jets.

Acknowledgements. The author thanks two anonymous reviewers for their insightful comments on the paper, which helped to improve the manuscript, and the editorial staff of Nonlinear Processes in Geophysics for their valuable work. Helpful discussions with Antonio Speranza, Valerio Lucarini and Renato Vitolo are gratefully acknowledged. The School of Science and Technology, the School of Advanced Studies of the University of Camerino and Fabio Marchesoni are kindly acknowledged for partially funding this publication.

Edited by: V. Perez-Munuzuri

Reviewed by: two anonymous referees

\section{References}

Allen, R. J., Sherwood, S. C., Norris, J. R., and Zender, C. S.: Recent Northern Hemisphere tropical expansion primarily driven by black carbon and tropospheric ozone, Nature, 485, 350-354, 2012.

Caballero, R., Pierrehumbert, R. T., and Mitchell, J. L.: Axisymmetric nearly inviscid circulations in non-condensing radiativeconvective atmospheres, Q. J. Roy. Meteorol. Soc., 134, 12691285, 2008.

Cessi, P.: angular momentum and temperature homogenization in the symmetric circulation of the atmosphere, J. Atmos. Sci., 55, 1997-2015, 1998.

Chen, G., Lu, J., and Sun, L.: Delineating the eddy-zonal flow interaction in the atmospheric circulation response to climate forcing:
Uniform SST warming in an idealized aquaplanet Model, J. Atmos. Sci., 70, 2214-2233, doi:10.1175/JAS-D-12-0248.1, 2013.

Dima, I. M. and Wallace, J. M.: On the seasonality of the Hadley cell, J. Atmos. Sci., 60, 1522-1527, 2003.

Fang, M. and Tung, K. K.: Time-dependent nonlinear Hadley circulation, J. Atmos. Sci., 56, 1797-1807, 1999.

Farrell, B. F.: Equable Climate Dynamics, J. Atmos. Sci., 47, 29862995, doi:10.1175/1520-0469(1990)047<2986:ECD>2.0.CO;2, 1990.

Frierson, D. M. W., Lu, J., and Chen, G.: Width of the Hadley cell in simple and comprehensive general circulation models, Geophys. Res. Lett., L18804, doi:10.1029/2007GL031115, 2007.

$\mathrm{Fu}, \mathrm{Q}$. and Lin, P.: Poleward shift of subtropical jets inferred from satellite-observed lower stratospheric temperatures, J. Climate, 24, 5597-5603, doi:10.1175/JCLI-D-11-00027.1, 2011.

Fu, Q., Johanson, C. M., Wallace, J. M., and Reichler, T.: Enhanced mid-latitude tropospheric warming in satellite measurements, Science, 312, 1179, doi:101126/science.1125566, 2006.

Gastineau, G., Le Treut, H., and Li, L.: Hadley circulation changes under global warming conditions indicated by coupled climate models, Tellus, 60, 863-884, doi:10.1111/j.16000870.2008.00344.x, 2008.

Gitelman, A. I., Risbey, J. S., Kass, R. E., and Rosen, R. D.: Trends in the surface meridional temperature gradient, Geophys. Res. Lett., 24, 1243-1246, 1997.

Greenwood, D. R. and Wing, S. L.: Eocene continental climates and latitudinal temperature gradients, Geology, 23, 1044-1048, 1995.

Held, I. M. and Hou, A. Y.: Nonlinear axially symmetric circulation in a nearly inviscid atmosphere, J. Atmos. Sci., 37, 515-533, 1980.

Hou, A. Y. and Lindzen, R. S.: The influence of concentrated heating on the Hadley circulation, J. Atmos. Sci., 49, 1233-1241, 1992.

Hu, Y. and Fu, Q.: Observed poleward expansion of the Hadley circulation since 1979, Atmos. Chem. Phys., 7, 5229-5236, doi:10.5194/acp-7-5229-2007, 2007.

Johanson, C. M. and Fu, Q.: Hadley cell widening: Model simulations versus observations, J. Climate, 22, 2713-2725, 2009.

Kang, S. M. and Polvani, L. M.: The interannual relationship between the latitude of the eddy-driven jet and the edge of the Hadley Cell, J. Climate, 24, 563-568, doi:10.1175/2010JCLI4077.1, 2011.

Kim, H.-K. and Lee, S.: Hadley cell dynamics in a primitive equation model, Part II: Nonaxisymmetric flow, J. Atmos. Sci., 58, 2859-2871, 2001.

Lindzen, R. S. and Hou, A. Y.: Hadley circulations for zonally averaged heating centered off the Equator, J. Atmos. Sci., 45, 24162427, 1988.

Liu, J., Song, M., Hu, Y., and Ren, X.: Changes in the strength and width of the Hadley Circulation since 1871, Clim. Past, 8, 11691175, doi:10.5194/cp-8-1169-2012, 2012.

Lu, J., Vecchi, G. A., and Reichler, T.: Expansion of the Hadley cell under global warming, Geophys. Res. Lett., 34, L06805, doi:10.1029/2006GL028443, 2007.

Lu, J., Chen, G., and Frierson, D. M. W.: Response of the zonal mean atmospheric circulation to El Niño versus global warming, J. Climate, 21, 5835-5851, doi:10.1175/2008JCLI2200.1, 2008. 
Lu, J., Deser, C., and Reichler, T.: Cause of the widening of the tropical belt since 1958, Geophys. Res. Lett., 36, L03803, doi:10.1029/2008GL036076, 2009.

Nguyen, H., Evans, A., Lucas, C., Smith, I., and Timbal, B.: The Hadley circulation in reanalyses: Climatology, variability, and change, J. Climate, 26, 3357-3376, 2013.

Polvani, L. M., Waugh, D. W., Correa, G. J. P., and Son, S.-W.: Stratospheric ozone depletion: the main driver of 20th century atmospheric circulation changes in the Southern Hemisphere, J. Climate, 24, 795-812, doi:10.1175/2010JCLI3772.1, 2011.

Santer, B. D., Thorne, P. W., Haimberger, L., Taylor, K. E., Wigley, T. M. L., Lanzante, J. R., Solomon, S., Free, M., Gleckler, P. J., Jones, P. D., Karl, T. R., Klein, S. A., Mears, C., Nychka, D., Schmidt, G. A., Sherwood, S. C., and Wentz, F. J.: Consistency of modelled and observed temperature trends in the tropical troposphere, Int. J. Climatol., 28, 1703-1722, 2008.

Schaller, N., Cermak, J., Wild, M., and Knutti, R.: The sensitivity of the modeled energy budget and hydrological cycle to $\mathrm{CO}_{2}$ and solar forcing, Earth Syst. Dynam., 4, 253-266, doi:10.5194/esd4-253-2013, 2013.

Schneider, E. K.: Axially symmetric steady-state models of the basic state for instability and climate studies. Part II. Nonlinear calculations, J. Atmos. Sci., 34, 280-296, 1977.

Schneider, E. K. and Lindzen, R. S.: Axially symmetric steady state models of the basic state of instability and climate studies, Part I: Linearized calculations, J. Atmos. Sci., 34, 253-279, 1977.

Seidel, D. J., Fu, Q., Randel, W. J., and Reichler, T. J.: Widening of the tropical belt in a changing climate, Nat. Geosci., 1, 21-24, 2008.
Sherwood, S. C., Meyer, C. L., Allen, R. J., and Titchner, H. A.: Robust tropospheric warming revealed by iteratively homogenized radiosonde data, J. Climate, 21, 5336-5352, 2008.

Staten, P. W., Rutz, J., Reichler, T., and Lu, J.: Breaking down the tropospheric circulation response by forcing, Clim. Dynam., 39, 2361-2375, doi:10.1007/s00382-011-1267-y, 2011.

Strong, C. and Davis, R. E.: Winter jet stream trends over the Northern Hemisphere, Q. J. Roy. Meteorol. Soc., 133, 2109-2115, 2007.

Tandon, N. F., Gerber, E. P., Sobel, A. H., and Polvani, L. M.: Understanding Hadley cell expansion versus contraction: Insights from simplified models and implications for recent observations, J. Climate, 26, 4304-4321, doi:10.1175/JCLI-D-1200598.1, 2013.

Titchner, H. A., Thorne, P. W., McCarthy, M. P., Tett, S. F. B., Haimberger L., and Parker, D. E.: Critically reassessing tropospheric temperature trends from radiosondes using realistic validation experiments, J. Climate, 22, 465-485, 2008.

Waliser, D. E., Shi, Z., Lanzante, J. R., and Oort, A. H.: The Hadley circulation: Assessing NCEP/NCAR reanalysis and sparse in situ estimates, Clim. Dynam., 15, 719-735, 1999.

Walker, C. C. and Schneider, T.: Response of idealized Hadley circulations to seasonally varying heating, Geophys. Res. Lett., 32, L06813, doi:10.1029/2004GL022304, 2005.

Walker, C. C. and Schneider, T.: Eddy influences on Hadley circulations: Simulations with an idealized GCM, J. Atmos. Sci., 63 3333-3350, 2006. 